\title{
Simulación de sistemas para la optimización del almacenamiento y despacho de los productos de gas natural
}

\author{
Rafael Segovia Castro \\ Ingeniería Industrial n. 27, 2009, ISSN 1025-9929, pp. 81-98
}

RESUmen: Tras el hallazgo del yacimiento de Camisea, con 13 TPC de reservas de gas natural y 480 millones de barriles de líquidos asociados, surgió la necesidad de contar con plantas para procesar dichos hidrocarburos en forma eficiente y cumplir con los clientes de forma oportuna. El objetivo de este trabajo es analizar la sección de almacenamiento y despacho en una planta de fraccionamiento de líquidos de gas natural ubicada en la costa peruana. A fin de realizar una evaluación cuantitativa dinámica en el tiempo de las mejoras propuestas, se procedió a diseñar una simulación que representara las operaciones tanto en la planta (producción) como en el terminal marino (carga de buques). Para esto se utilizó el programa de simulación AweSim ${ }^{\circledR} 3.0$ (Student Version) del fabricante Symix Systems, Inc.

Palabras clave: hidrocarburos / líquidos de gas natural / Camisea / almacenamiento / simulación de sistemas / fraccionamiento

\section{Systems simulation for optimizing storage and loading of natural gas products}

ABSTRACT: With the discovery of the Camisea fields, comprising 13 TCF of natural gas reserves and 480 millon barrels of associated liquids, the need for plants which could process these hydrocarbons efficiently and fulfill customers' requeriments appeared. The aim of this paper is to analyze the storage and dispatch in a fractionating plant of natural gas liquids located in the Peruvian coast. In order to perform a cuantitative and dynamic evaluation on time of the proposed enhancements, a simulation was designed which represented the operations both in the plant and in the marine loading platform. The simulation program AweSim ${ }^{\circledR} 3.0$ (Student Version) by Symix Systems, Inc., was used for this purporse.

Keywords: hidrocarbons / natural gas liquids / Camisea / storage / systems simulation / fractionation 


\section{INTRODUCCIÓN}

Con la firma del contrato de explotación el año 2000, entre el Gobierno peruano y el consorcio liderado por la empresa Pluspetrol, se inició el desarrollo de los yacimientos de gas natural más importantes del país. Con $13 \mathrm{TPC}^{1}$ de reservas de gas natural y 480 millones de barriles de líquidos asociados, dichos yacimientos son, sin duda, una fuente importante de energía para el Perú y de ingresos económicos significativos, debido a la exportacion de los excedentes de GLP ${ }^{2}$ y nafta no consumidos localmente, y en un futuro cercano del gas natural licuefactado, que será exportado a México. Asimismo, las importaciones de combustibles líquidos han disminuido, mejorando la balanza comercial de hidrocarburos.

Con el descubrimiento de estas nuevas reservas aparece la necesidad de contar con una industria de hidrocarburos más madura, con plantas capaces de procesar efectivamente el gas y el petróleo, con el fin de obtener el mayor valor agregado posible.

Figura 1

Balanza comercial de hidrocarburos

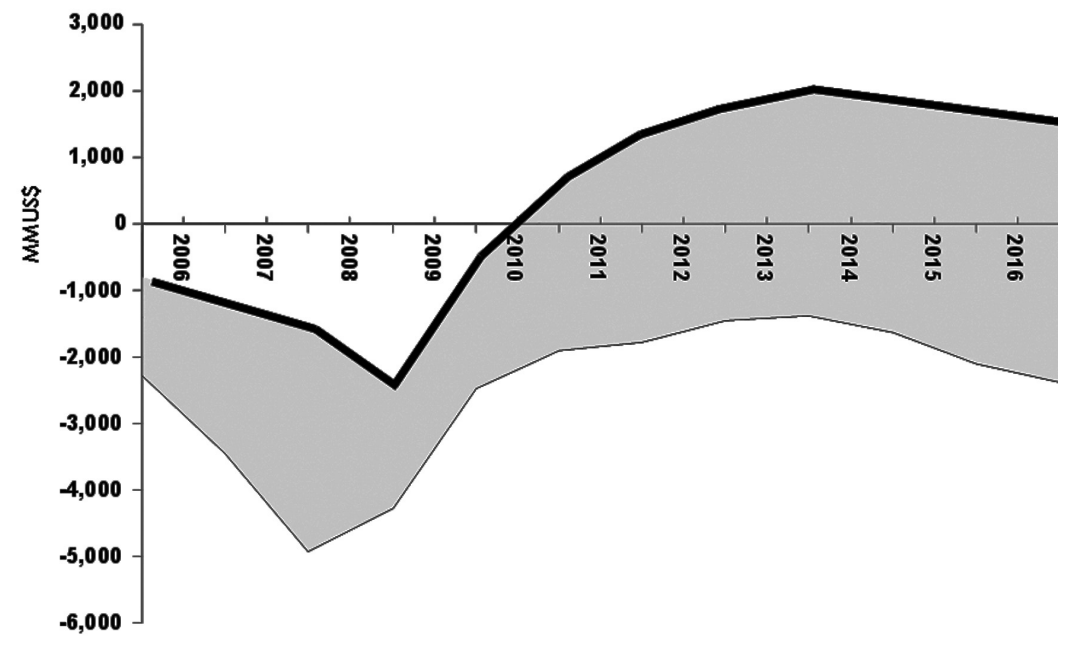

1 Trillones de pies cúbicos, medida volumétrica de gas natural, equivalente a $28,3168 \mathrm{x}$ $10^{9} \mathrm{~m}^{3}$.

2 Gas licuado de petróleo. 
El objetivo del presente trabajo es estudiar la sección de almacenamiento y despacho en una planta de fraccionamiento de líquidos de gas natural, operaciones que son críticas para el adecuado funcionamiento de la planta. Se analizará la situación actual de los tanques y se propondrán mejoras para optimizar el almacenamiento en la planta. Asimismo, se evaluarán los factores relacionados con el despacho en buques, a fin de contar con una logística más eficiente. Se espera obtener una mejora conjunta en estas dos secciones para poder operar con mayor capacidad y cumplir con el cliente de manera oportuna.

\section{CADENA DE VALOR DEL GAS NATURAL}

Para tener una idea más clara de la cadena de valor del gas natural se procederá a explicar sus etapas. Se divide en dos grandes grupos: el upstream, que comprende las actividades relacionadas con la exploración y explotación de los pozos de hidrocarburos; y el downstream, que incluye la refinación o tratamiento de los hidrocarburos a fin de obtener los combustibles requeridos por el mercado, los cuales serán comercializados mediante buques, camiones cisterna o ductos.

Figura 2

Cadena de valor del gas natural

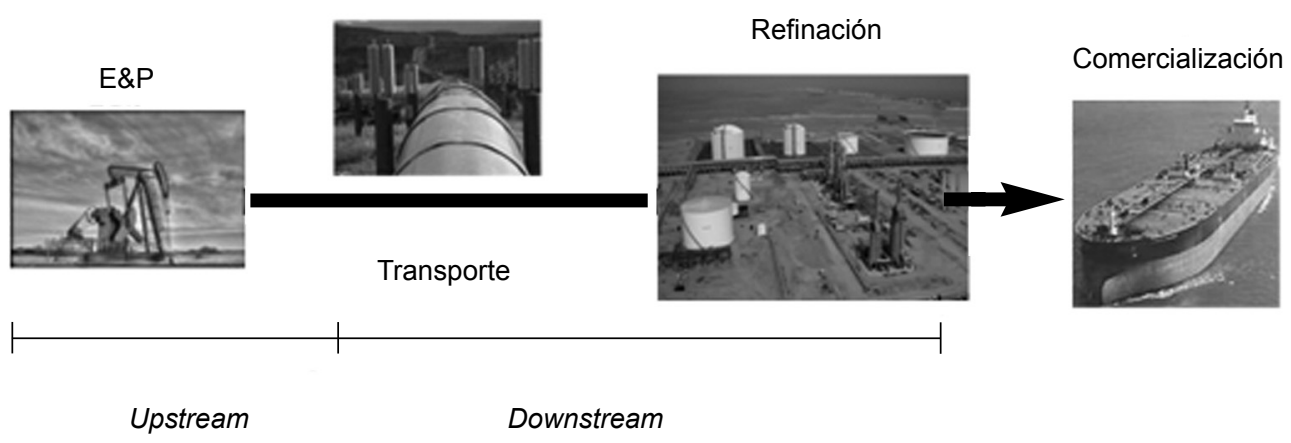




\section{CASO DE ESTUDIO}

En esta ocasión, el caso de estudio es una planta de fraccionamiento de líquidos de gas natural localizada en la costa peruana, la que recibe la materia prima desde pozos de gas asociados ubicados en Camisea, en el oriente del país. Los líquidos de gas natural son transportados desde la selva hasta la planta mediante un poliducto.

Las etapas del proceso de la planta de fraccionamiento comprenden dos unidades principales: una unidad de fraccionamiento y una unidad de separación. La planta recibe los líquidos de gas natural (LGN) provenientes de los yacimientos, y luego de las destilaciones correspondientes se obtienen cuatro productos: propano y butano en la unidad de fraccionamiento; nafta y diésel en la unidad de separación.

Figura 3

Diagrama de entradas y salidas de la planta de fraccionamiento

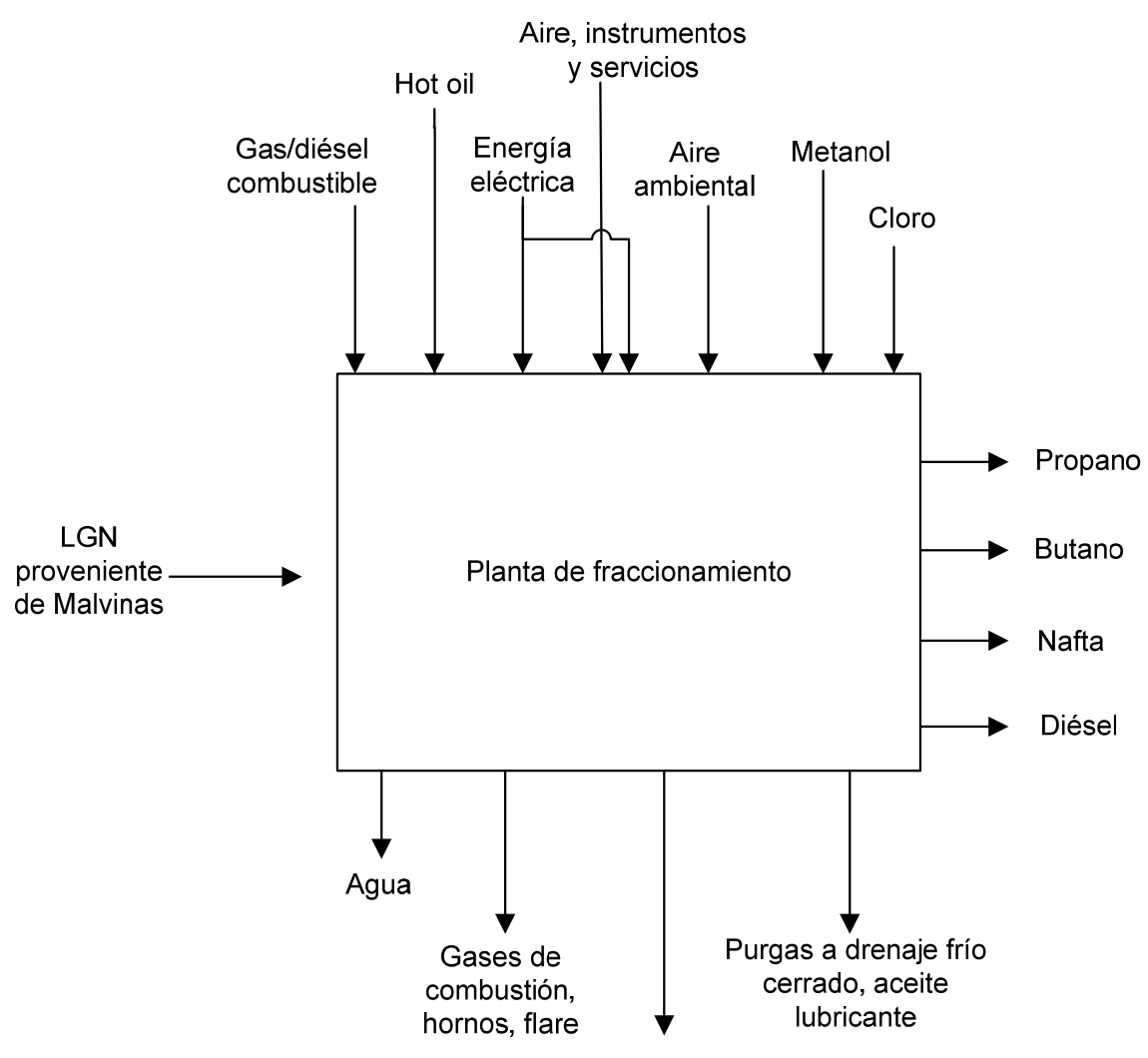


Luego de hacer el análisis de la planta, se determinó que el principal problema referido al almacenamiento está relacionado con la poca capacidad de los tanques, así como al reducido número de estos por producto. La limitada capacidad de almacenamiento se aprecia al comparar la producción diaria y la frecuencia de los embarques. La tabla 1 muestra las producciones promedio diarias de cada producto, los lotes embarcables y la frecuencia de los buques.

Tabla 1

Información sobre los productos de la planta

\begin{tabular}{lccc}
\hline Producto & $\begin{array}{c}\text { Producción } \\
\text { diaria }\end{array}$ & $\begin{array}{c}\text { Lote por } \\
\text { embarque }\end{array}$ & $\begin{array}{c}\text { Frecuencia de } \\
\text { embarques }\end{array}$ \\
\hline Propano & $1.000 \mathrm{TM}$ & $2.200 \mathrm{TM}$ & 5 días \\
Butano & $540 \mathrm{TM}$ & $1.850 \mathrm{TM}$ & 5 días \\
Nafta & $17.000 \mathrm{Bbl}$ & $280.000 \mathrm{Bbl}$ & 17 días \\
Diésel & $2.500 \mathrm{Bbl}$ & $40.000 \mathrm{Bbl}$ & 15 días \\
\hline
\end{tabular}

* Abreviación de barril (medida de volumen y productos de petróleo), equivalente a 42 galones americanos o 158,99 litros.

Hay ocasiones en que los buques se retrasan y esto ocasiona que los productos no puedan ser evacuados. Un buque puede retrasarse por demoras ocasionadas en puertos anteriores, como problemas al cruzar el Canal de Panamá (de ser el caso) o desperfectos de la máquina. Adicionalmente, el clima puede jugar en contra, creando condiciones poco seguras para el amarre de los buques en la plataforma de carga, dilatando los cargamentos. Por otro lado, el principal problema relacionado con el despacho de productos por buque está referido a las bombas de embarque de diésel.

En la figura 4 se puede apreciar que el rate o caudal de carga promedio en un embarque de diésel es de 2.800 barriles (limitado por planta) por hora. Sin embargo, el flujo de carga de los buques es de hasta 5.000 barriles por hora, por lo que la diferencia en el rate de carga se materializa en demoras pagadas por la planta. Esta realidad se muestra en la figura 4. 
Figura 4

Demoras debido a bajo rate de carga

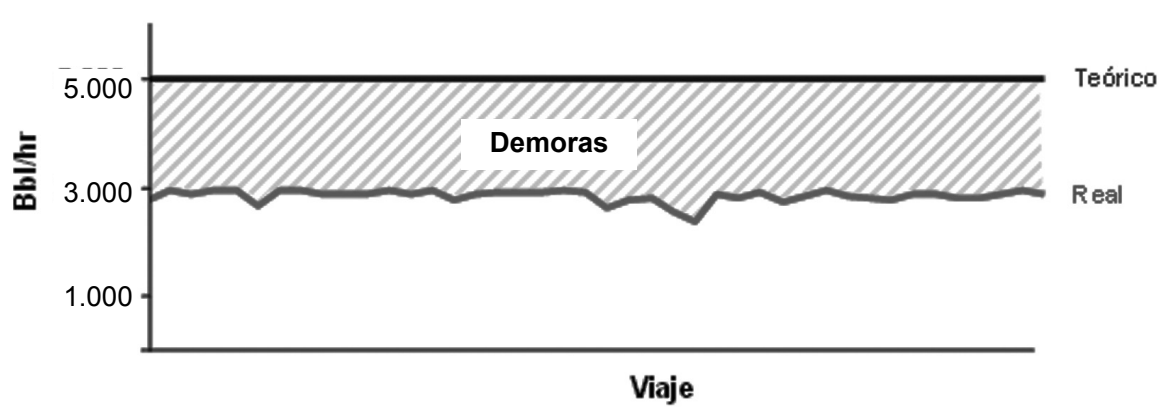

\section{SOLUCIONES PROPUESTAS PARA LOS PROBLEMAS IDENTIFICADOS}

Para solucionar el problema del almacenamiento se plantearon diversas opciones, como las siguientes:

- Construcción de nuevos tanques de almacenamiento.

- Contar con buques que funcionen como almacenamiento flotante.

- Interconexión con otras plantas que cuenten con capacidad libre de almacenamiento.

- Cambio en lotes y frecuencia de los buques.

La solución más sólida es la ampliación de la capacidad de almacenamiento de la planta de fraccionamiento, a través de la construcción de nuevos tanques, ya que ayudaría a evitar el "sobrestock" (exceso de inventario en almacenamiento), posibilitaría la medición de productos para la venta de manera estática y, no menos importante, sería una inversión con mucho beneficio cuando se empiece a explotar gas y líquidos de los pozos de Camisea que aún no están en producción, así como para cuando empiece a funcionar el proyecto Perú LNG, ${ }^{3}$ en el 2010 .

3 El Proyecto de Perú LNG consiste en la construcción de una planta de licuefacción de gas natural, la cual recibirá el gas de Camisea y lo transformará a estado líquido para exportarlo principalmente a Norteamérica. 
Para el análisis se escogieron los tanques de nafta y diésel, porque estos productos son los más difíciles de controlar en la actualidad, pues se venden según la modalidad FOB, ${ }^{4}$ en la que el cliente pone el buque. Estas naves no siempre llegan a tiempo o están disponibles, poniendo en peligro de sobrestock a la planta. En el caso del GLP, se cuenta con un buque, destinado a la venta CIF ${ }^{5}$ que es controlado por la planta, la cual organiza sus días de carga a fin de manejar inventarios razonables. Adicionalmente, el GLP puede ser distribuido en camiones cisterna, ya que la planta cuenta con tres islas de carga de camiones, donde, en caso de emergencia y trabajando las 24 horas del día, se podría despachar este producto, dando así cierto margen de operación.

Del estudio económico realizado de la instalación de un tanque nuevo de diésel con las mismas características que los tanques actuales, utilizando un $\mathrm{CPPC}^{6}$ para los cálculos del 15\%, se determinó que el proyecto de un nuevo tanque de diésel tiene un VAN de US\$ 2.059.822 y el periodo de recuperación es inferior al año. La TIR no es representativa en este caso, por tener un valor demasiado grande. Se concluye que el proyecto del nuevo tanque de diésel sería viable. También el proyecto del tanque de nafta es atractivo, pues cuenta con un VAN positivo y una TIR por encima del $15 \%$. Además, posee un periodo de recuperación reducido. Por lo dicho, se recomienda llevar a cabo también este proyecto.

Para resolver los problemas relacionados con las bombas de diésel y mejorar el despacho de productos se determinó que la mejor opción consistía en la adquisición de nuevas bombas, con un rate mayor de carga. El costo total de las bombas, incluyendo la instalación, sería de US\$415.000. Si se compara el rate de carga actual del buque con el de tierra se tiene un déficit de 2.300 barriles por hora, equivalente a 8 días de demoras al año, que multiplicado por el costo de demora anual daría un pago de demoras de US\$152.865. Esto se evitaría implementando el proyecto de nuevas bombas.

4 FOB (Free On Board): Modalidad de venta en la cual el comprador es responsable del transporte del producto

5 CIF (Cost, Insurance and Freight): Modalidad de venta en la cual el vendedor es responsable del transporte del producto.

6 Costo de capital promedio. Porcentaje utilizado para calcular rentabilidad de los proyectos. 
Luego de analizar los embarques de diésel durante un año, se pudo determinar que había buques de nafta y de GLP que debieron anclar y esperar a que la carga del diésel terminara. De haber cargado el buque de diésel con mayor rapidez se habría liberado más pronto el terminal, reduciendo dichas esperas que incurrieron en demoras.

Asimismo, se debe considerar la situación cuando el tanque de alguno de los productos esté en situación crítica de sobrestock. El hecho de que la carga del diésel demore aproximadamente ocho horas más por embarque, dilata el ingreso de otros buques que podrían decrecer el stock de los tanques y reducir así situaciones de parada de planta o reducciones de carga. Al igual que en el estudio anterior, se utilizó un CPPC de 15\%, concluyéndose que el proyecto de compras de nuevas bombas para el cargamento de diésel es viable al tener un VAN igual a US\$1.127.109.

\section{SIMULACIÓN DE SISTEMAS}

Según la definición de Robert E. Shannon, ${ }^{7}$ la simulación es el "[...] proceso de diseñar y desarrollar un modelo computarizado de un sistema o proceso y conducir experimentos con este modelo con el propósito de entender el comportamiento del sistema y/o evaluar varias estrategias con las cuales se puede operar el sistema".

Los recientes avances en las metodologías de simulación y la gran disponibilidad de de diversos tipos de software existentes en el mercado han hecho que la técnica de simulación sea una de las herramientas más ampliamente usadas en el análisis de sistemas. Thomas H. Naylor ${ }^{8}$ ha sugerido que un estudio de simulación es muy importante para la ingeniería porque presenta las siguientes ventajas:

- A través de un estudio de simulación se puede investigar el efecto de cambios internos y externos del sistema, al hacer alteraciones en el modelo y observar los efectos de esas alteraciones en el comportamiento del sistema.

- Una observación detallada del sistema que se está simulando puede conducir a un mejor entendimiento de este, y por consi-

7 Shannon, Robert E. La simulación de los sistemas. El arte y ciencia, los principios de Englewood, 1975.

8 Naylor, Thomas H., citado en Omar, Enrique. "Simulación". 
guiente a sugerir estrategias que mejoren su operación y su eficiencia.

- La técnica de simulación puede ser utilizada para experimentar con nuevas situaciones, sobre las cuales se tiene poca o ninguna información. A través de esta experimentación se puede anticipar mejor a posibles resultados no previstos.

\subsection{Uso de la simulación de procesos}

A fin de realizar una evolución cuantitativa dinámica en el tiempo de las mejoras propuestas, se procedió a diseñar una simulación que representara las operaciones tanto en la planta (producción) como en el terminal marino (carga de buques). Para esto se utilizó el programa de simulación AweSim ${ }^{\circledR} 3.0$ (Student Version) del fabricante Symix Systems, Inc.

AweSim es un software de simulación de propósito específico que se basa en simulación por procesos. Los modelos se definen sobre la base de entidades que fluyen por el sistema. Cada entidad tendrá asociados ciertos atributos (estáticos o dinámicos), como tiempo de llegada, tiempo en que comienza a ser atendido, edad, etcétera. Adicionalmente, el modelo puede manejar dos tipos de variables: para entidades y globales.

Los elementos básicos de AweSim son la red y el control. En la red se modela el flujo de las entidades a través de los procesos y está compuesta por un conjunto de nodos interconectados. Existen varios tipos de nodos, cada uno realiza una función específica cuando pasa una entidad por él. En el control se definen las entradas y se asignan los atributos que supervisan la simulación. La figura 5 muestra la lógica seguida en el diseño de la simulación relacionada con la carga de buques.

Las informaciones que se obtienen de esta simulación son las siguientes: las paradas de planta (debido a sobrestock $\mathrm{u}$ otros factores), las demoras de cada buque, esperas por falta de producto o terminal ocupado, números de viajes y otros datos relacionados con las operaciones marítimas.

Con el fin de poder comparar el estado actual de la planta con el que resultaría al implementar las recomendaciones de este trabajo, se ejecutó la simulación utilizando dos escenarios. El primero contempla 


\section{Figura 5}

Lógica seguida en la simulación propuesta.

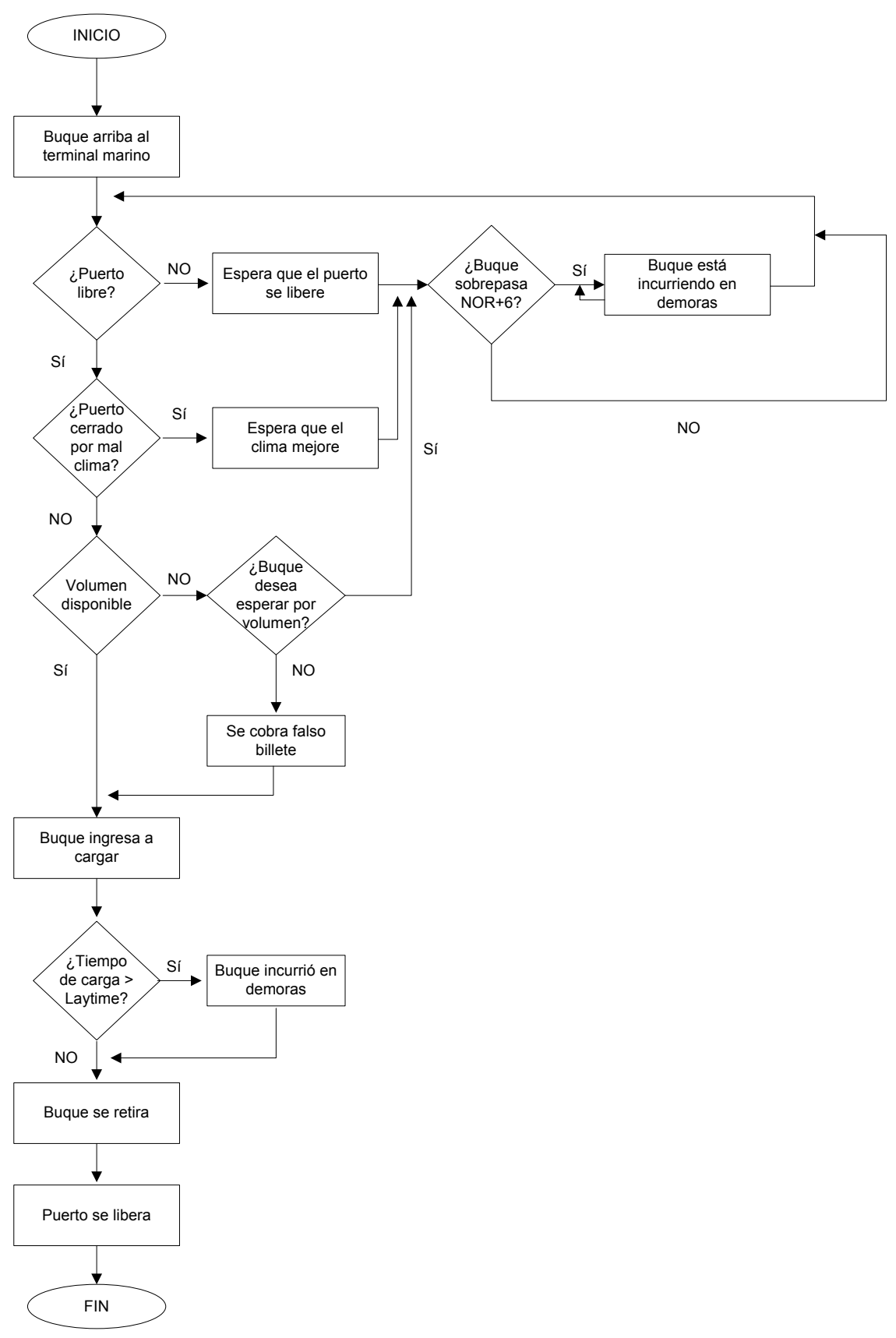


la situación actual de la planta, que es justamente la que se desea mejorar. Se analizarán las diversas variables y datos pero se hará hincapié en los temas relacionados con el almacenaje y las demoras en las cargas. En el segundo escenario se evalúa si los cambios propuestos en este trabajo, como la construcción de tanques, el cambio de bombas de diésel, entre otros, son posibles y valen la pena.

\subsection{Escenario A: Estado actual de la planta y terminal}

A partir de los resultados obtenidos de la simulación del escenario A se pueden realizar las siguientes observaciones: el buque de diésel es el que tiene más demoras (más de cuatro veces del buque que le sigue). Esto se debe básicamente a los retrasos sistemáticos que ocurren en todos los cargamentos por el bajo rate de carga de las bombas de tierra.

Figura 6

Demoras promedio de los diversos buques

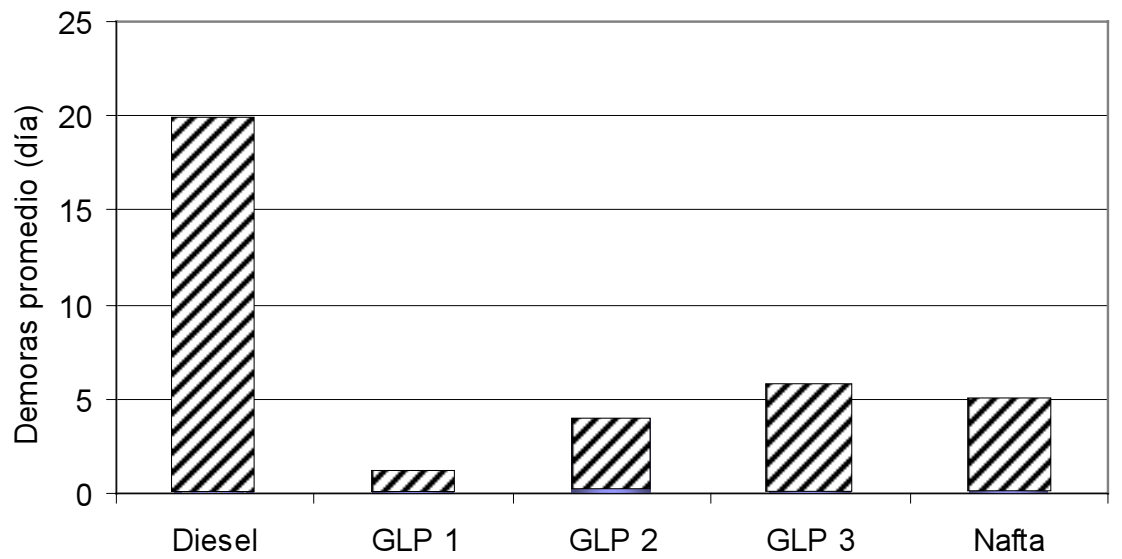

Las paradas de planta por sobrestock también resultan interesantes. Los tanques que aparecen en situación crítica son el de nafta y el de propano, como se muestra en la figura 7. 
Figura 7

Paradas de planta debido a sobrestock de tanques

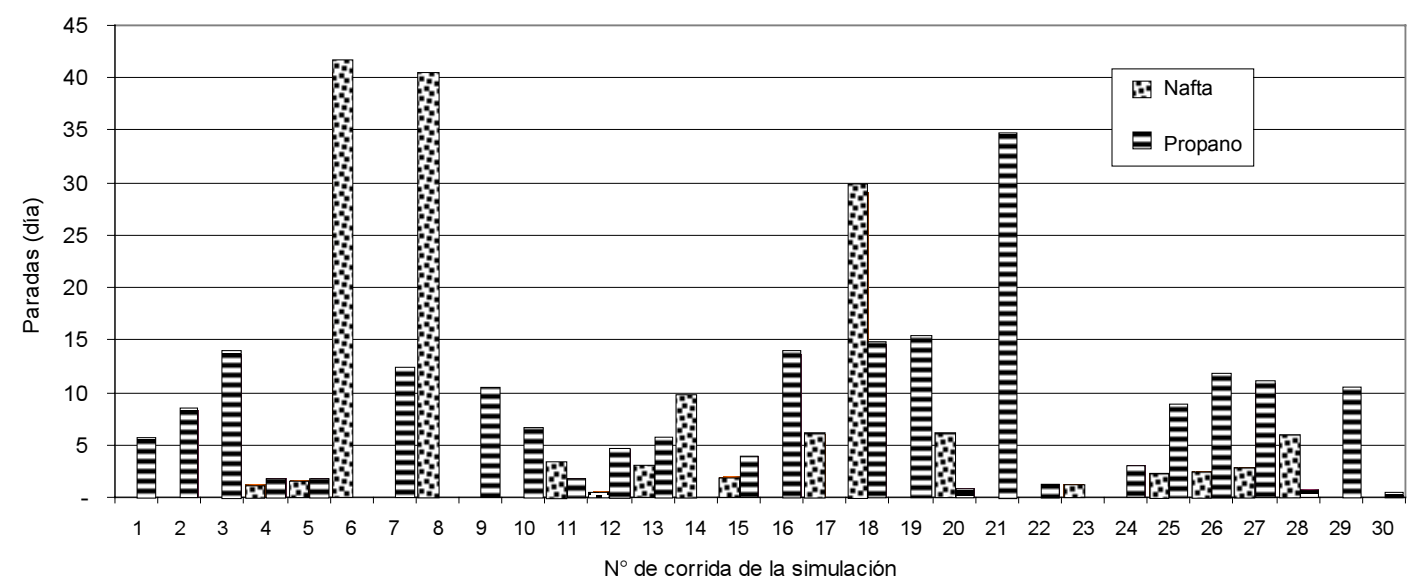

También se pueden extraer los tiempos de carga promedio de cada buque, tiempo que incluye el tiempo de carga, espera y demora.

Figura 8

Tiempos totales de carga

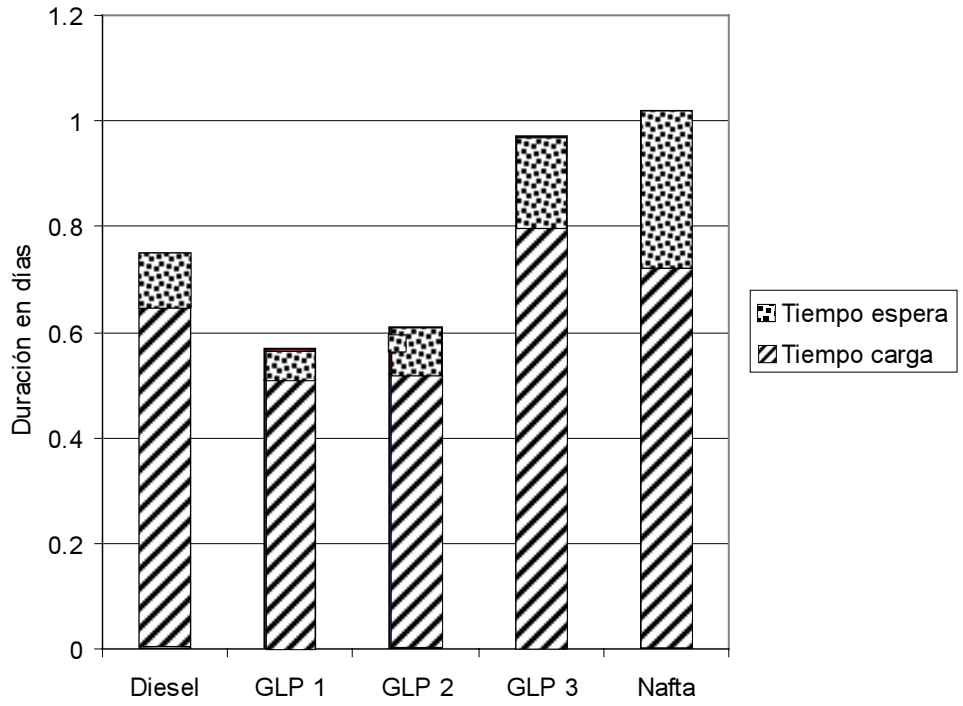




\subsection{Escenario B: Estado modificado con implementación de las mejoras}

Para este escenario se puede observar que el buque de diésel continúa con la mayor cantidad de demoras; sin embargo, es un valor mucho menor que el del escenario anterior.

Figura 9

Demoras promedio totales de cada buque

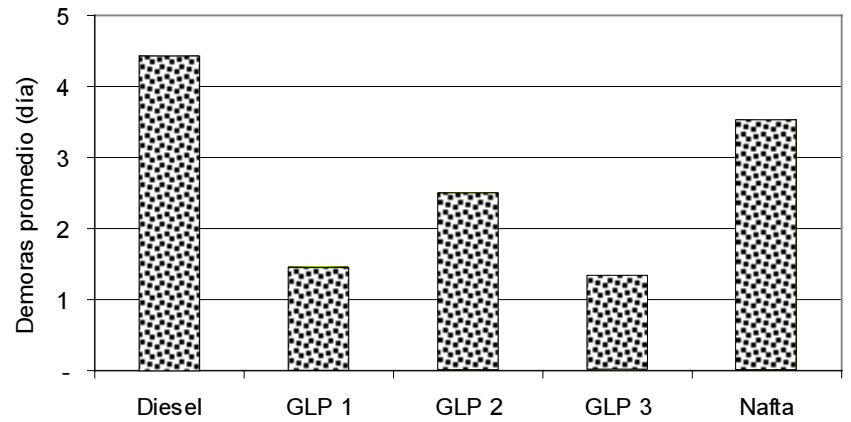

No hay paradas de producción debido al sobrestock; por ello, se podría concluir que la opción de aumentar la capacidad de almacenamiento ha sido acertada. En el caso de los tiempos totales de carga tenemos:

Figura 10

Tiempos totales de carga

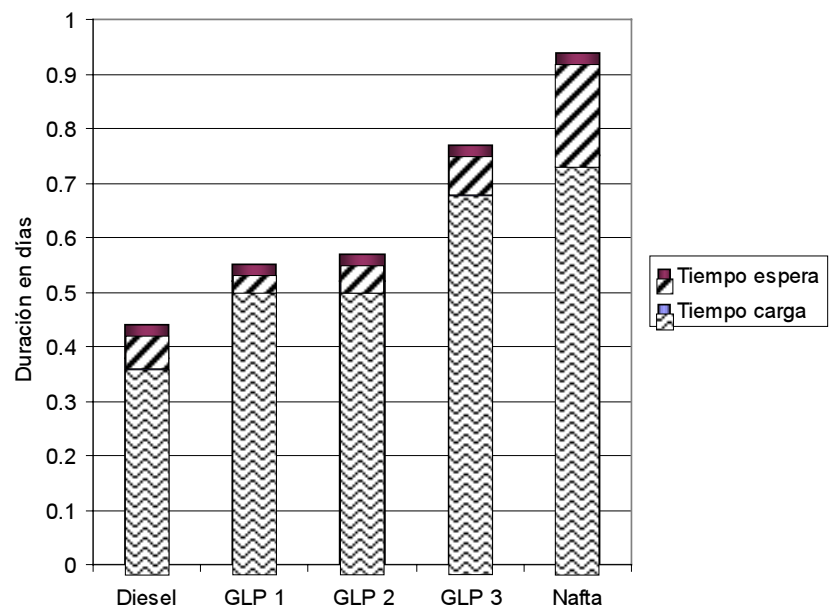


Al comparar ambos escenarios se concluye que en el escenario B:

- El tiempo total promedio de estadía (carga + esperas) de un buque en el terminal de carga disminuye en un $11 \%$ (de 0,695 a 0,618 días).

- El tiempo de esperas (incluyendo demoras) del buque de diésel en un viaje se reduce en $34 \%$ (0,102 a 0,068 días). En el tiempo total de simulación, y contando todos los buques de este producto, la reducción es del orden del $300 \%$.

- Los tiempos de carga de todos los buques también se reducen.

- Las demoras de todos los buques disminuyen.

- Ya no hay paradas de planta por sobrestock.

- El recurso "espacio", que representa al terminal marino de carga, disminuye su tiempo promedio de uso, por lo que el terminal se liberaría más rápido y así más buques podrían ser atendidos durante ese lapso.

Los gráficos que se presentan a continuación muestran estas conclusiones. En el tiempo total de estadía de cada buque se puede apreciar una reducción en todos los casos, también hay importantes cambios en el caso del diésel y el buque GLP 3 (buque más grande de GLP). Si bien en los otros buques la reducción no parece significativa, a largo plazo estos tiempos suman y sin duda afectan económicamente a la empresa. Las demoras promedio totales también disminuyen, reduciéndose casi todas en más de un 50\% como mínimo.

Figura 11

Comparación de tiempo total de estadía

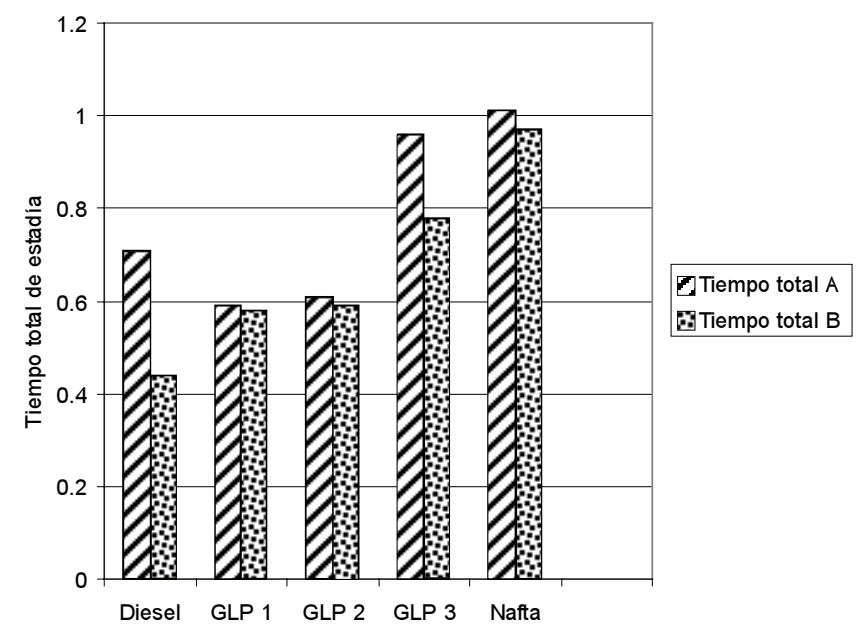


Figura 12

Comparación de demoras promedio totales de cada buque

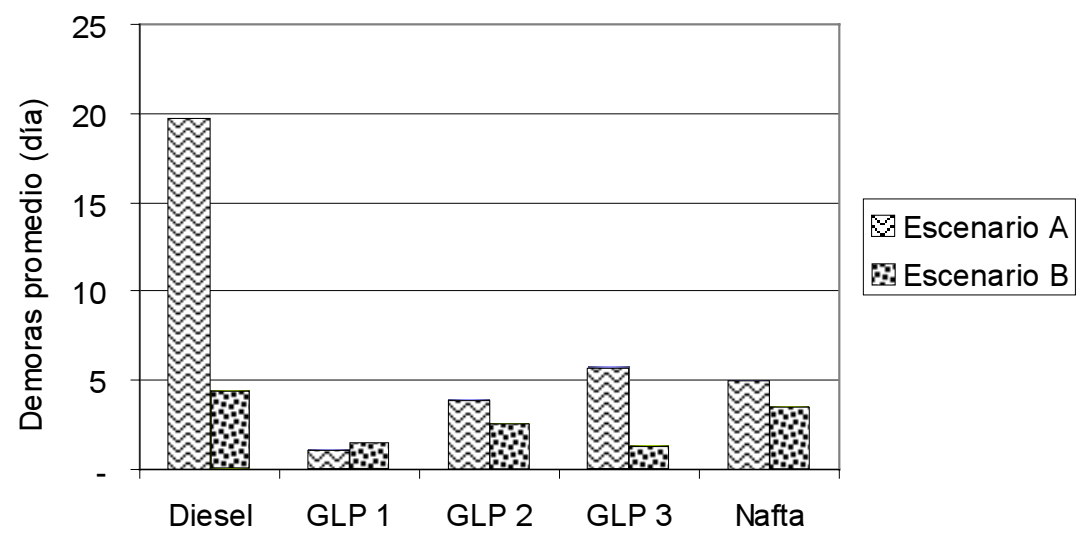

Como se mencionó, el recurso terminal de carga se libera más rápido, por lo que se podría utilizar más veces, es decir, una mayor cantidad de buques podrían amarrar y cargar al año en el terminal marino.

Figura 13

Ocupación promedio del terminal de carga

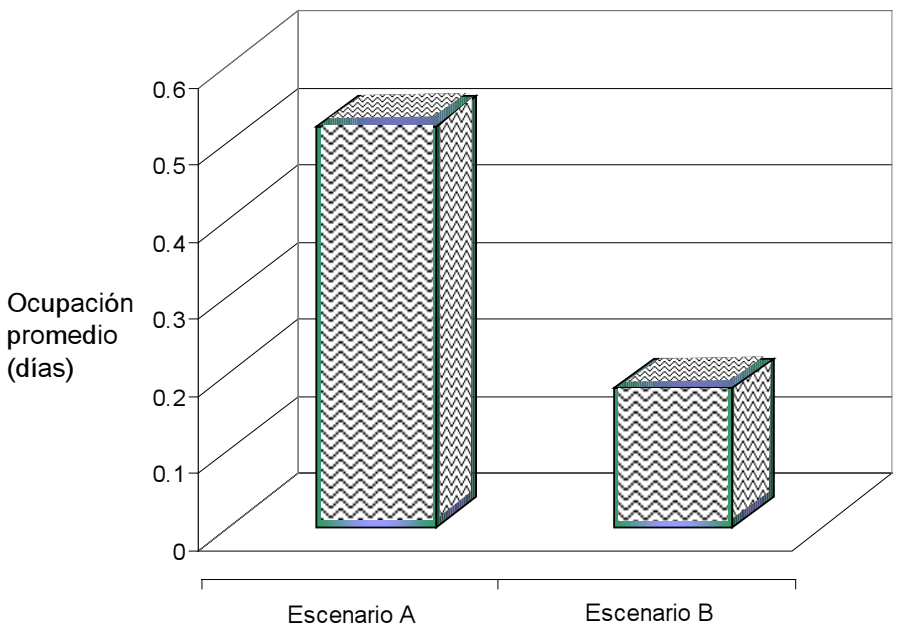




\subsection{Bombas de diésel}

La adquisición de nuevas bombas con mayor rate de carga permitirá el ahorro por el pago de demoras. Utilizando los datos obtenidos de la simulación del AweSim llegamos al siguiente número:

20 días (Escenario A) - 5 días (Escenario B) = 15 días x US\$20.000/día

$=$ US\$300.000 que se ahorrarían al año.

Figura 14

Demoras promedio del buque de diésel

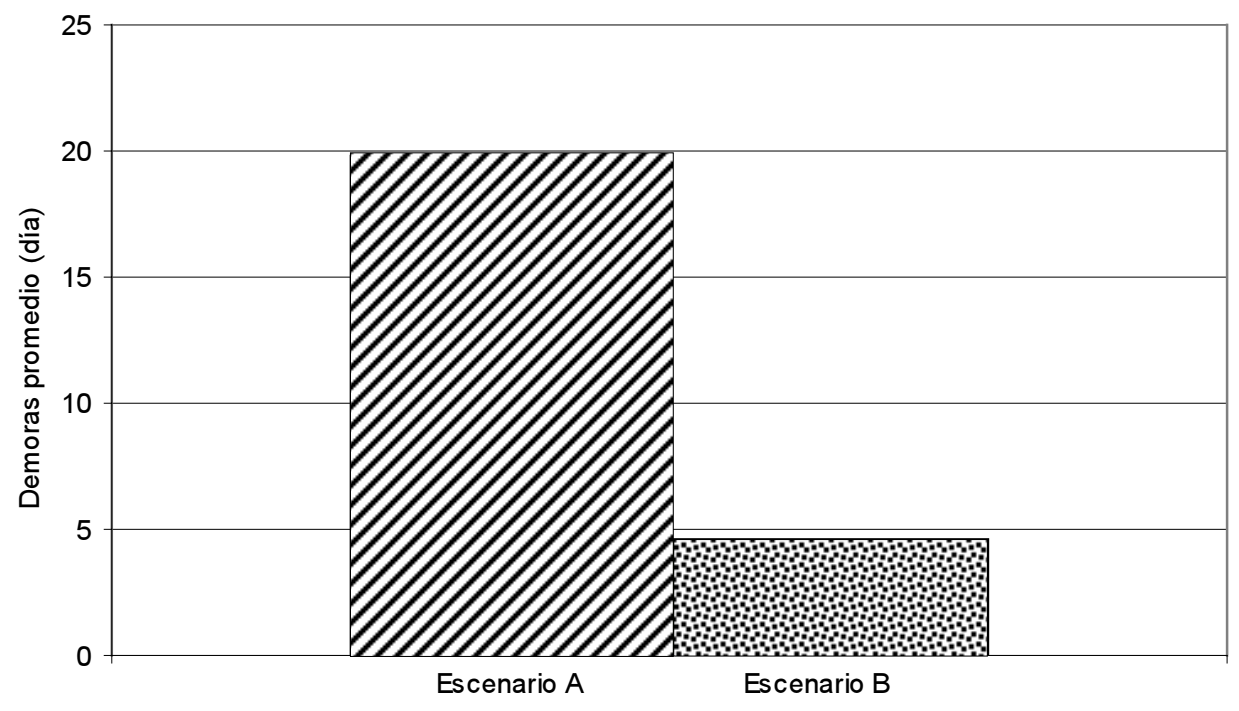

Adicionalmente a este cálculo se pudo determinar que hubo buques de nafta y GLP que debieron permanecer anclados a la espera de que el buque de diésel libere el terminal de carga, así como la situación en que el tanque de alguno de los productos esté en situación crítica de sobrestock. El hecho de que la carga de diésel demore más tiempo por embarque dilata el ingreso de otros buques, que podrían disminuir el stock de los tanques y eliminar así situaciones de parada de planta o reducciones de carga. 


\section{CONCLUSIONES}

- La simulación de sistemas es una herramienta muy útil en la evaluación de proyectos de mejora, porque permite, de manera más económica y científica, manipular la realidad a fin de determinar cómo se comportaría el sistema ante los cambios. La simulación hará posible realizar estos cambios virtualmente.

- El principal problema de la planta de fraccionamiento de Pisco, según lo observado hasta la fecha, es la capacidad de almacenamiento. La mejor solución vislumbrada desde el punto de vista económico y pragmático es construir más tanques.

- El hecho de contar con más tanques de almacenamiento permitirá evitar los casos de sobrestock, contar con tanques estáticos que facilitan las mediciones y fiscalizaciones, tener una mayor flexibilidad de operación, sobre todo cuando se requiera mantenimiento, y permitirá, especialmente, estar preparados para el inicio de la segunda etapa de Camisea.

- Asimismo, el bajo rate del diésel que causa demoras se solucionaría con unas bombas que permitirían, además, cargar buques más grandes. El cambio a bombas con un mayor rate de carga posibilitaría ahorrar unos US\$300.000. 


\section{BIBLIOGRAFÍA}

Enraf, B.V. "The Art of tank gauging" [en línea]. <http://www.enraftankgauging.com/>. [Consulta: julio del 2009.]

Gas Processors Suppliers Association (GPSA). Engineering data book. 12. a edición. Tulsa, Ok, 2004.

International Maritime Organization (IMO). "International Convention for the Control and Management of Ships' Ballast and Sediments, 2004" [en línea]. <http://www.imo.com/>. [Consulta: julio del 2009.]

Matthews, Philip. Advanced chemistry. Cambridge, UK: Cambridge University Press, 1992.

Ministerio de Energía y Minas [en línea]. <http://www.minem.gob.pe/>. [Consulta: julio del 2009.]

Morales, F. Gestión de proyectos. Madrid: Instituto Superior de la Energía (ISE), 2007.

Omar, Enrique. "Simulación” [en línea]. <http://html.rincondelvago/ simulacion.html>. [Consulta: julio del 2009].

Pedrosa, J. y F. Subirà. "El inventariado en tanques (I y II)". Ingeniería Química 360 y 361. Madrid: Alción, 1999.

Pemex. Norma de Referencia NRF-015-PEMEX-2003. México: Comité de Normalización de Petróleos Mexicanos y Organismos Subsidiarios, 2003.

Pieltain, M. y J. Fernández-Cuesta. Modalidades de fletamento. Madrid: Instituto Superior de la Energía (ISE), 2007.

Proyecto Camisea [en línea]. <http://www.camisea.com.pe/>. [Consulta: julio del 2009.]

Reglamento de Seguridad para el Almacenamiento de Hidrocarburos. D.S. N 36-2003-EM. Lima, 2003.

Romano, Silvia Daniela. Tanques de almacenamiento de hidrocarburos. Buenos Aires: Universidad de Buenos Aires, Facultad de Ingeniería (Fiuba), 2004.

Russo, G.; Galcerán, M. y C. Rodríguez. Curso de medición de gas. Buenos Aires, 2004.

Shannon, Robert E. La simulación de los sistemas. El arte y ciencia, los principio de Englewood. Nueva jersey: Prentice-Hall, 1975. 